\title{
Imaging Li-Ion Battery Material with Low Voltage Backscattered Electrons - comparison of a Field Emission SEM Crossbeam540/Merlin with the DELTA SEM
}

\author{
$\underline{\text { U. Golla-Schindler }^{1}}{ }^{*}$, I. Wacker ${ }^{2,3}$, B. Schindler ${ }^{4}$, T. Bernthaler ${ }^{1}$, G. Schneider ${ }^{1}$ and R.R. Schröder ${ }^{2,3}$
}

1. Materials Research Institute (IMFAA), Aalen University, Aalen, Germany.

2. Centre for Advanced Materials (CAM), University Heidelberg, Heidelberg, Germany.

3. Cryo Electron Microscopy, BioQuant, University Hospital Heidelberg, Germany.

4. Carl Zeiss Microscopy, Oberkochen, Germany.

*ute.golla-schindler@hs-aalen.de

In numerous consumer electronics and electric vehicles lithium-ion batteries (LIB) have become one of the most prevalent electric storage systems. LIBs are complex systems to understand and their degradation processes during operation are not clear up to now. There the generation of surface layers like the SEI (solid electrolyte interface) on the anode side and the SPI (semi permeable interface) on the cathode side play an important role for the functionality and lifetime of a battery. The inspection of such surface layers is difficult and scanning electron microscopy is one of the key technologies to visualize those layers. This study was performed using two instruments, a Crossbeam 540/Merlin SEM column equipped with an inlens energy selecting backscattered (EsB) detector with a retarding grid in front of the detector. The second instrument was a novel low-voltage SEM prototype developed in the framework of the DELTA-project based in Heidelberg [1]. This instrument allows correction of spherical and chromatic aberrations and has a newly designed DELTA Detector ${ }^{\circledR}$. This detector employs two retarding grids improving energy resolution and has an increased detection efficiency due to an enlarged detection solid angle. Both detectors enable energy-filtered imaging, where solely electrons with energies above the grid voltage contribute to the image. Lower landing energy decreases the penetration depth and increases the spatial resolution for material contrast information but there is a limit at $1 \mathrm{keV}$. Below $1 \mathrm{keV}$ landing energy the material contrast relationship - the higher the signal the higher the atomic number of the investigated material - is not valid any more [2]. Therefore, our study was performed at $1 \mathrm{keV}$ landing energy. The sample was a cathode foil of a commercially available graphite/NMC $\left(\mathrm{Li}\left[\mathrm{Co}_{1 / 3} \mathrm{Ni}_{1 / 3} \mathrm{Mn}_{1 / 3}\right] \mathrm{O}_{2}\right)$ pouch cell of a cycled battery. Figure 1 shows images taken with the EsB detector $(\mathrm{a}, \mathrm{c}, \mathrm{e}, \mathrm{g})$ and the DELTA detector ${ }^{\circledR}(\mathrm{b}, \mathrm{d}, \mathrm{f}, \mathrm{h})$ with changing grid voltages of $0 \mathrm{~V}$ $(\mathrm{a}, \mathrm{b}),-50 \mathrm{~V}(\mathrm{c}, \mathrm{d}),-700 \mathrm{~V}(\mathrm{e}, \mathrm{f})$ and $-900 \mathrm{~V}(\mathrm{~g}, \mathrm{~h})$. They show conductive additives covered by a coating, which is created when cells degenerate. This coat reduces the sharpness and resolution of the particles. The image information changes for low grid voltages from topographical information (a) and (b) to material contrast information in $(\mathrm{g})$ and $(\mathrm{h})$. By definition, secondary electrons (SEs) are those with energies below $50 \mathrm{eV}$, with the main peak located around $10 \mathrm{eV}$. Due to this they can only leave the bulk material at surface near regions, thus creating the "edge effect" - bright rims visible at the edges of the particles marked in (a) and (b) with arrowheads. With increasing grid voltage, the SEs are repelled and solely BSE contribute to the image. Thus, the information depth decreases with increasing grid voltage reducing the information depth to the sub $\mathrm{nm}$ range. This enables the detection of solely the lowloss electrons (e-h). There are significant differences between EsB and DELTA images: The precipitates with high atomic number may be detected in the EsB but are certainly resolved in the DELTA images (white arrows). Additionally, the numbers of detectable precipitates rise and their sharpness improves in the Delta images from (f) to (h) (red arrows). The DELTA SEM opens new avenues of application and offers unique information about high-resolution material contrast. 
[1] R.R. Schröder et al., Microscopy and Microanalysis 24 (2018) p. 626

[2] U. Golla-Schindler et al., Micron 113 (2018) p. 10-19

[3] R. Böngeler et al., Scanning 15 (1993) p. 1-18

[4] We thank the Center for Advanced Materials and ZEISS GmbH for the DELTA images.
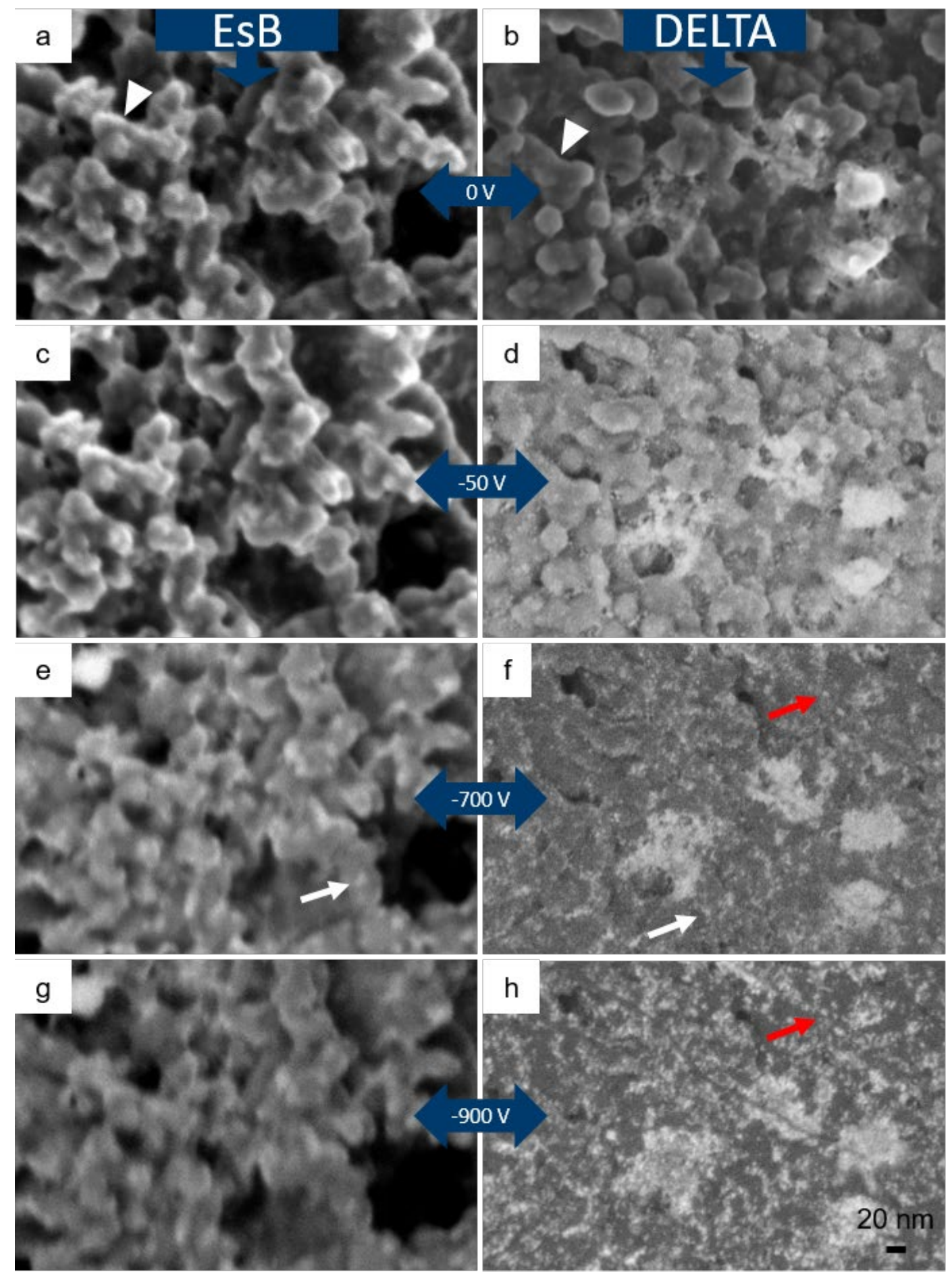

Figure 1. Image series of conductive additive of a cycled NMC cathode foil. Recorded (a, c, e, g) with the EsB detector (Crossbeam 540) and (b, d, f, h) with the DELTA detector ${ }^{\circledR}$ (DELTA-SEM). Retarding grid voltage $(\mathrm{a}, \mathrm{b})-10 \mathrm{~V},(\mathrm{c}, \mathrm{d})-50 \mathrm{~V},(\mathrm{e}, \mathrm{f})-700 \mathrm{~V}$ and $(\mathrm{g}, \mathrm{h})-900 \mathrm{~V}$. 